being a very disabling symptom, the variables that predict it are still unknown.

Objectives: To identify potential factors associated with fatigue.

Methods: Cross-sectional study. 60 patients were included, patients were being followed up in the rheumatology outpatient clinic of Hospital Clínico San Carlos, Madrid, Spain. Data were collected between July 2018 and January 2019. All patients met the ACR/EULAR 2010 criteria and they were in treatment with Biological agents or Targeted Synthetic DMARDs. Main variable: Fatigue was assessed by the Bristol Rheumatoid Arthritis Fatigue Multidimensional Questionnaire (BRAF-MDQ) and the Bristol Rheumatoid Arthritis Fatigue Numerical Rating Scale (BRAF-NRS). Covariables: Sociodemographic, clinical, Disease-related variables and treatment. Statistical analysis. A descriptive analysis was carried out for the different variables. To identify factors independently associated to BRAF-MDQ a multivariable linear regression was applied. Results were expressed as $\beta$ with their corresponding $95 \% \mathrm{Cl}$. A value of $p<0.05$ was accepted as statistically significant.

Results: A total of 60 patients, comprising of 53 females $(88 \%)$ and 7 males $(12 \%)$ with a mean age of $56.45 \pm 11.89$ years and mean disease duration of $14.11 \pm 7.79$ years were evaluated. RF was positive in $60 \%$ of patients. $65 \%$ of the patients were in active on work and $23 \%$ were retired. Regarding comorbidities, 43\% had dyslipidemia, 42\% hypertension, $17 \%$ hypothyroidism and $15 \%$ depression. The DAS28-ESR and SDAI scores of the patients were $2.68 \pm 0.86,8.56 \pm 5.96$ respectively. The rest of the clinical, treatment characteristics, and the scores of the fatigue instruments used in the study are shown in Table 1. Physical dimension was the most affected according to patients, who perceive an average severity and affectation, and insufficient coping. The factors affecting the total BRAF-MDQ was evaluated in multivariate analyze Table 2. We found that patients with Disability $(p<0.0001)$ and those in treatment with Non anti-TNF ( $p$ 0.048), experienced more fatigue while a positive RF ( $p$ $0.022)$ decreases overall fatigue.

Conclusion: Fatigue is a substantial symptom in RA patients and should be evaluated on the daily clinical practice. The results of our study indicated that disability and type of treatment were the dominant factors in the experience and degree of fatigue. Disease activity (DAS28 and SDAI) was not associated to fatigue. The evaluation and management of fatigue should be one of the objectives in the treatment of patients with RA

Table 1. Characteristics of the sample

\begin{tabular}{lc}
\hline Variable & Descriptive statistics \\
\hline Glucocorticoids mg/day, mean \pm SD & $4.86 \pm 1.65$ \\
Treatment, $\mathbf{n}(\%)$ & $47(78.33)$ \\
Conventional DMARDs & $40(66.67)$ \\
Anti TNF & $16(26.67)$ \\
Non-Anti TNF & $4(6.67)$ \\
Synthetic target DMARD (Jakinibs). & \\
HAQ (0-3) mean \pm SD & $0,86 \pm 7,7$ \\
VAS pain mean \pm SD & $39.3 \pm 24.27$ \\
Sleep quality self-report, $\mathbf{n}(\%)$ & $23(39.66)$ \\
Good & $35(60.34)$ \\
Poor & \\
BRAF-MDQ (0-70), mean \pm SD & $21.95 \pm 15.01$ \\
Physical (0-22) & $10.6 \pm 5.49$ \\
Living (0-21) & $5.08 \pm 5.04$ \\
Cognitive (0-15) & $3.3 \pm 3.38$ \\
Emotional (0-12) & $2.96 \pm 2.75$ \\
BRAF-NRS, mean \pm SD & \\
Fatigue severity (0-10) & $4.75 \pm 2.33$ \\
Effect of fatigue (0-10) & $4.8 \pm 2.43$ \\
Coping with fatigue (0-10) & $3.73 \pm 2.29$ \\
\hline
\end{tabular}

Table 2. Multivariate analyses of Total-BRAF-MDQ

\begin{tabular}{lccccc}
\hline Total BRAF & Coef. & $\begin{array}{l}\text { Std. } \\
\text { Err. }\end{array}$ & $\mathbf{t}$ & $\mathbf{p}$ & IC 95\% \\
\hline Age & -0.041 & 0.141 & -0.29 & 0.769 & -0.3250 .242 \\
Sex & -6.933 & 4.985 & -1.39 & 0.170 & -16.9383 .071 \\
FR positive & -9.589 & 3.467 & -2.77 & 0.008 & $-16.546-$ \\
& & & & & 2.631 \\
Non Anti- & 7.348 & 3.621 & 2.03 & 0.048 & 0.08214 .614 \\
TNF & & & & & \\
HAQ $>\mathbf{1 . 1}$ & 15.863 & 3.290 & 4.82 & 0.000 & 9.25922 .467 \\
\hline
\end{tabular}

Disclosure of Interests: None declared

DOI: 10.1136/annrheumdis-2019-eular.7177

\section{AB0247 INSIGHTS INTO ADHERENCE AND PATIENT-INITIATED MONOTHERAPY FOR RHEUMATOID ARTHRITIS VIA A GLOBAL SURVEY OF PATIENTS, CAREGIVERS AND PHYSICIANS}

James Galloway ${ }^{1}$, Ara Dikranian ${ }^{2}$, Cheryl L. Koehn ${ }^{3}$, David C. Gruben ${ }^{4}$, John Woolcott ${ }^{5}$, Sander Strengholt $6 .{ }^{1}$ King's College and King's College Hospital, London, United Kingdom; ${ }^{2}$ Cabrillo Center for Rheumatic Disease, San Diego, CA, United States of America; ${ }^{3}$ Arthritis Consumer Experts, Vancouver, BC, Canada; ${ }^{4}$ Pfizer Inc, Groton, CT, United States of America; ${ }^{5}$ Pfizer Inc, Collegeville, PA, United States of America; ${ }^{6}$ Pfizer Inc, Capelle aan den IJssel, Netherlands

Background: Treatment satisfaction and adherence remain issues affecting outcomes for patients (pts) with rheumatoid arthritis (RA). A better understanding of the reasons for non-adherence, and differences between pt, caregiver and healthcare professional (HCP) views on adherence, may improve decision-making and outcomes.

Objectives: To survey pts, caregivers and HCPs, in order to investigate pt behaviours and attitudes, and communications between pts, caregivers and HCPs regarding adherence to RA therapy.

Methods: Surveys were conducted online by Harris Poll between Oct and Nov 2018, among pts/caregivers and HCPs from Australia, Brazil, Canada, Germany, Japan, Spain, Taiwan, UK and US. The pt survey included pts aged $\geq 18$ years with a diagnosis of RA who had taken conventional synthetic disease-modifying antirheumatic drugs (csDMARDs) with biological (b)DMARDs in the past 3 years. The caregiver survey included adults aged $\geq 18$ years who lived with/provided care to a spouse/family member with RA and were involved in $>2$ care activities for a pt with RA who had taken csDMARDs with bDMARDs in the past 3 years. The HCP survey included responses from licensed rheumatologists (orthopaedists in Japan) who saw $\geq 10$ pts with moderate/severe RA per month, $\geq 10 \%$ of whom had been prescribed a bDMARD. A postweight was applied to raw data to adjust for the relative adult population size of each country vs the total for all 9 countries surveyed.

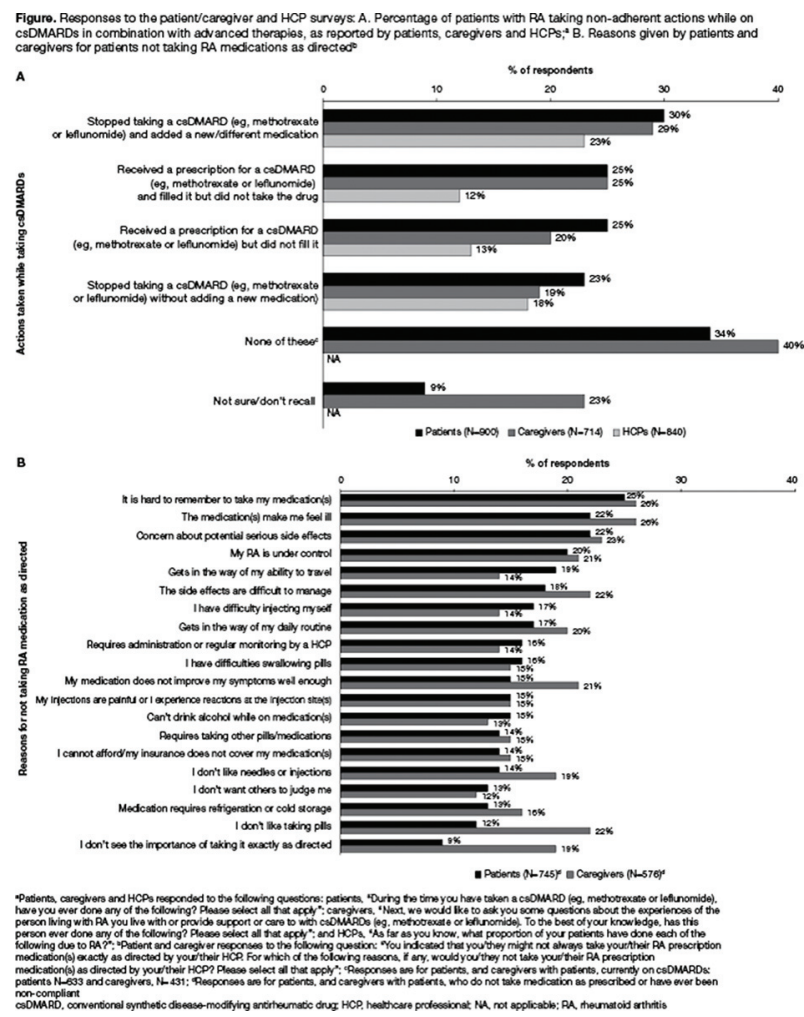

Figure 1

Results: In total, 900 pts, 714 caregivers and 840 HCPs responded to the surveys. Overall, $50 \%$ of both pts and caregivers reported that pts always took their current RA prescription medications exactly as directed However, $67 \%$ of pts reported that they had not always adhered to dosing regimens (most common deviations: skipping doses [45\%]; extending time between doses [44\%]; taking other medications without HCP direction $[38 \%]$; taking less than the prescribed dose [34\%]; dose splitting [31\%]; and stopping medications altogether [28\%]). Among HCP 
respondents, 98\% reported asking pts about adherence to RA medication, even though $61 \%$ felt there was no way of knowing if pts were taking medications as prescribed and, overall, HCPs responded that $64 \%$ of pts did not always take RA medication as prescribed. While $87 \%$ of HCPs agreed that bDMARDs were most effective when taken in combination with csDMARDs and $92 \%$ reiterated this to pts, $62 \%$ were concerned that pts take bDMARDs without csDMARDs. Pts and caregivers reported that pts often stopped taking csDMARDs; however, HCPs underestimated the number of non-adherent pts (Figure A). The most common reason given by pts and caregivers for non-adherence to RA medication was difficulty in remembering to take medication; however, concerns about feeling ill and potential side effects were also common (Figure B). Despite this, $47 \%$ of pts and $51 \%$ of caregivers responded that pts often don't share all side effects caused by their RA medication with HCPs, while $38 \%$ of HCPs believed that pts did not inform them of all side effects.

Conclusion: This study from a large, multinational cohort of pts with RA, caregivers and HCPs describes medication adherence patterns. Pts on bDMARDs often did not take their background csDMARDs; however, the extent of pt non-adherence was underestimated by HCPs. These results highlight gaps in communication and understanding of non-adherence between pts, caregivers and HCPs.

Acknowledgement: Study sponsored by Pfizer Inc. Medical writing support was provided by Anthony G McCluskey, PhD, of CMC Connect and funded by Pfizer Inc. Disclosure of Interests: James Galloway Consultant for: Pfizer Inc, Ara Dikranian Consultant for: AbbVie, Pfizer Inc, Speakers bureau: AbbVie, Pfizer Inc, Cheryl L Koehn Shareholder of: Arthritis Consumer Experts, Grant/research support from: Canadian Institutes of Health Research, Arthritis Research Canada, St. Paul's Hospital, Employee of: Arthritis Consumer Experts, David C Gruben Shareholder of: Pfizer Inc, Employee of: Pfizer Inc, John Woolcott Shareholder of: Pfizer Inc, Employee of: Pfizer Inc, Sander Strengholt Shareholder of: Pfizer Inc, Employee of: Pfizer Inc DOI: 10.1136/annrheumdis-2019-eular.761

\section{AB0248 DISEASE ACTIVITY IS ASSOCIATED WITH FATIGUE IN THE FOLLOW-UP OF PATIENTS WITH RHEUMATOID ARTHRITIS}

Rocío Violeta Gamboa Cárdenas ${ }^{1}$, Manuel F. Ugarte-Gil ${ }^{2}$, Cristina

Reategui Sokolova ${ }^{3}$, Luz Hipólito ${ }^{4}$, Mariela Medina ${ }^{3}$, Claudia Elera-Fitzcarrald ${ }^{5}$, Victor Pimentel Quiroz ${ }^{3}$, Paola Alejandra Zeña Huancas ${ }^{3}$, Francisco Zevallos ${ }^{3}$, Samira Garcia-Hirsh ${ }^{3}$, Luciana Giil ${ }^{3}$, Zoila Rodriguez Bellido ${ }^{3}$, Cesar

Pastor Asurza ${ }^{3}$, Risto Perich Campos ${ }^{3} .{ }^{1}$ Hospital nacional Guillermo Almenara es Salud, Rheumatology, Lima, Peru; ${ }^{2}$ Universidad Científica del Sur, Rheumatology, Lima, Peru; ${ }^{3}$ Hospital Nacional Guillermo Almenara Essalud, Rheumatology, Lima, Peru; ${ }^{4}$ Hospital Emergencias Grau EsSalud, Rheumatology, Lima, Peru;

${ }^{2}$ Universidad Científica del Sur, Rheumatology, Lima, Peru

Background: Chronic fatigue negatively impacts on quality of life in Rheumatoid Arthritis (RA) patients $(1,2)$, does not allow optimal clinical results, being an negative predictor for clinical remission $(3,4)$.

Objectives: The objective was to determine whether disease activity correlates with increased fatigue in the follow-up of a RA patients.

Methods: Prospective study of the RA-Almenara cohort (criteria ACR87) ACR-EULAR2010). Patients were included with at least two evaluations (half-yearly). Patients with fibromyalgia, major depression and/or anxious depressive disorder, chronic neuropathic pain or active systemic manifestations (including interstitial lung disease) were excluded. Disease activity was evaluated with SDAI (Simple Disease Activity Index) and fatigue with FACIT questionnaire (Functional Assessment of Chronic Illness Therapyfatigue). A generalized estimation equation model was used to determine the association between SDAI and FACIT, at each visit according with two models of analyses. Model 1 considered SDAI value as a linear variable; Model 2 considered each category of SDAl (high, moderate and low) activity, using remission as reference. Multivariable analyses were adjusted by possible confounders: gender, age at diagnosis, instruction, socio-economic level (Graffar), disease duration, tobacco, ACPA level, disability (MDHAQ), use of conventional (c) and biologic(b) DMARDs and corticosteroids (current use, past or non-use).

Results: Four hundred and twenty patients were included, 372 (88.6\%) women, age at diagnosis was 44.1 (13.2) years, disease duration 17.7 (11.6) years. At the baseline $4.2 \% 13.6 \%, 41.0 \%$ and $41.2 \%$ were in remission, low, moderate and high activity respectively; 40\% were using corticosteroids, $256(61 \%)$ cDMARDs and $25(6 \%)$ bDMARDs. Basal SDAl was 23.7 (20.4) and FACIT 17.2 (8.8). We analyzed 1233 follow up visits [2.2 (1.2) visits per patient]. Multivariablee analysis: In model 1 were associated with FACIT: SDAI [B: -0.03 (IC95\%: - 0.06- $-0.01 ; \mathrm{p}=0.013$ ] and MHAQ [B: -9.55 (IC95\%: - 10.55- -0.54), $\mathrm{p}=<0.001]$. In model 2, were associated with FACIT: the categories high [B: -4.92 (IC95\%: -6.87 - 2.7); $p<0.001]$, moderate [B: -3.75 (IC95\% : -5.70- -1.89), $\mathrm{p}<0.001]$ and low [B: $-2.22(95 \% \mathrm{Cl}$ : $-4.05--0.39) ; p=0.018$ ] disease activity by SDAl, in addition with MHAQ [B: -9.11 (IC95\%: - 10.11- -8.11), $p<0.001]$ and tobacco use [B: $-3.37(95 \% \mathrm{Cl}:-6.56--0.18, \mathrm{p}=0.039)$ ]

Conclusions: Higher disease activity correlates with worse fatigue scores, at each follow-up visits of patients with RA

\section{REFERENCE:}

[1] Balsamo. Isr. Med. Assoc. J. 2014, 16, 57-60. 2. Alomari. Sci. World J. 2012:580863. doi: 10.1100/2012/580863. 3. Roodenrijs. Ann Rheum Dis. 2018 Sep 7. pii: annrheumdis-2018-213687. 4 Uhlig. J Rheumatol. 2016;43(4):716-23

Disclosure of Interests: None declared

DOI: 10.1136/annrheumdis-2019-eular.5625

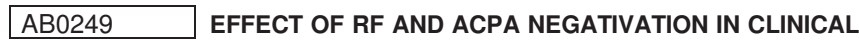 RESPONSE IN RA PATIENTS UNDER BIOLOGIC THERAPY}

Sara Ganhão ${ }^{1}$, Raquel Ferreira ${ }^{1}$, Bruno Miguel Fernandes ${ }^{1}$, Salomé Garcia ${ }^{1}$, Eva Mariz ${ }^{1}$, Miguel Bernardes ${ }^{1,2}$, Lúcia Costa ${ }^{1}{ }^{1}$ Centro Hospitalar e Universitário de São João, Department of Rheumatology, Oporto, Portugal; ${ }^{2}$ Faculty of Medicine of Oporto University, Oporto, Portugal

Background: Both rheumatoid factor (RF) and antibodies against cyclic citrullinated peptide (ACPA) are regarded as serological markers of Rheumatoid Arthritis (RA), being well recognized as diagnostic and prognostic tools. However, their potential role in the disease's monitoring and clinical response is still under debate.

Objectives: To assess the effect of RF and ACPA negativation in clinical response to biologic therapy in RA patients.

Methods: Longitudinal retrospective study of RA patients treated with biologic therapy as first line option. Demographic and clinical data were collected at baseline and at 24 months follow-up, including: RF and ACPA status (negative or positive), ESR, CRP, DAS28 4v ESR, CDAI, SDAI, $H A Q$, EULAR and ACR response. RF was considered positive if $\geq 30$ $\mathrm{U} / \mathrm{ml}$ and ACPA if $\geq 7 \mathrm{U} / \mathrm{ml}$. SPSS statistics 22.0 was used for statistical analysis.

Results: 169 patients were included with mean $( \pm S D)$ age of $50.7 \pm$ 10.6 years and median disease duration (min-max) of $10.2(0.69-39.4)$ years. The majority were female $(84 \%)$. At baseline $160(94.7 \%)$ were positive for RF and $166(98.2 \%)$ were positive for ACPA. 77 (45.6\%) turned negative for RF and/or ACPA at a median time of 20.3 months after biologic therapy begining. 63 out of 169 patients become negative for RF (37.3\%) and 17 patients for ACPA (10.1\%). 52 (30.6\%) patients were treated with etanercept, $39(22.9 \%)$ adalimumab, $27(15.9 \%)$ rituximab, 19 (11.2\%) tocilizumab; 16 infliximab (9.4\%); 14 (8.2\%) golimumab; $1(0.6 \%)$ certolizumab and $1(0.6 \%)$ anakinra. The mechanism of action of the drug didn't differ between patients who became seronegative for RF and/or ACPA and those who remained seropositive $(70.2 \%$ under anti-TNF $\alpha$ agents vs $73.9 \%$ for the other biologics). Most of the patients in the first group began adalimumab $(32.5 \%)$ and most of the patients in the latter began etanercept (34.8\%). Demographic characteristics like age, sex, disease duration, and extraarticular manifestations were comparable in both groups. They weren't comparable in terms of smoking habits $(p=0.014)$ : just $3(3.9 \%)$ current smokers in those who became negative for RF and/or ACPA vs 17 (18.5\%) among those who remained seropositive. Nevertheless, there weren't statistically significant differences in $\triangle \mathrm{DAS} 284 \mathrm{~V}$ ESR, $\triangle \mathrm{ERS}, \triangle \mathrm{CRP}, \triangle \mathrm{CDAl}, \triangle \mathrm{SDAl}, \triangle \mathrm{HAQ}$, EULAR or ACR response at 24-months between patients with negativation of at least one antibody and those who remained seropositive.

Conclusion: In our sample, change of antibody status wasn't predictor of better response to biologic therapy. Therefore the results did not support the association between the persistence of RF or ACPA and the lack of effectiveness of biologic therapy at 2 years of treatment, but further studies are needed.

\section{REFERENCE:}

[1] Alexandra B. et al. Prospective cohort study of effects of infliximab on rheumatoid factor, anti-cyclic citrullinated peptide antibodies and antinuclear antibodies in patients with long-standing rheumatoid arthritis; Joint Bone Spine 76 (2009) 248e253.

Disclosure of Interests: None declared

DOI: 10.1136/annrheumdis-2019-eular.5294 